\title{
Human papillomavirus vaccination: where to now?
}

\author{
S M Garland $d^{1,2,3,4}$
}

'Department of Microbiology and Infectious Diseases, The Royal Women's Hospital, Parkville, Australia ${ }^{2}$ Department of Obstetrics and Gynaecology, University of Melbourne, Parkville, Australia ${ }^{3}$ Infectious Disease and Microbiology, Murdoch Childrens Research Institute University of Melbourne, Parkville, Australia ${ }^{4}$ Department of Microbiology, Royal Children's Hospital, Parkville, Australia

\section{Correspondence to}

Professor Suzanne M Garland, Department of Microbiology and Infectious Diseases, Royal Women's Hospital, Locked Bag 300, Parkville 3052, Australia; suzanne.garland@thewomens. org.au

Accepted 11 August 2011

\section{(2) UNLOCKA}

This paper is freely available online under the BMJ Journals unlocked scheme, see http://sti. bmi.com/site/about/unlocked. xhtml

\section{INTRODUCTION}

Extraordinary developments have occurred since molecular and epidemiological tools have proven that oncogenic human papillomaviruses (HPVs) cause virtually all cervical cancers, as well as a proportion of other anogenital cancers (vulvar, vaginal, anal, penile) and some oropharyngeal cancers (particularly tonsillar). ${ }^{12}$ In addition, lowrisk HPV types 6 and 11 are the causative agents of the majority of genital warts, ${ }^{3}$ a benign but very common sexually transmitted disease, estimated to occur in up to $10 \%$ of the population ${ }^{4}$ and with an estimated annual burden of new cases worldwide of 30 million.

With the development of viral-like particles and the relatively short translation to phase 3 clinical vaccine trials showing that prophylactic HPV vaccines targeting the two most common causes of cervix cancer, HPV 16 and 18, are safe, immunogenic and efficacious, ${ }^{5-7}$ we now observe the outcome of these vaccines implemented as public health tools in those countries that can afford such programmes. Where coverage has been high to the appropriate target population, reduction in disease has already been seen for those HPV-related diseases with the shortest incubation periods. For example, in Australia the quadrivalent $\operatorname{HPV}(6,11$, 16, 18) vaccine was started in April 2007 in a comprehensive, national, government-funded, school-based, ongoing programme for girls 12 years of age, with a catch-up program to 26 years of age until December 2009. In this real-life situation, a statistically significant reduction has already been reported in genital warts of almost $60 \%$ in young women of vaccine-eligible age. ${ }^{8}$ These findings are most likely related to the catch-up component of the programme, rather than the school-based programme. However, with time and continued good vaccine coverage, one would expect a change in sexual health clinics, where treatment of genital warts currently makes up a large component of the work. Genital warts not only carry a huge financial burden, but also cause substantial psychosocial burden and, in immunocompromised patients such as those with HIV infection, can be recalcitrant to standard treatment and difficult to manage.

Moreover, although initial registration of HPV vaccines in Australia allowed vaccination of boys 10-15 years of age, there is currently no government or health insurance subsidy for males: consequently only a very small proportion of males have received vaccination. Yet, with the high coverage in young women of around $70 \%$, a significant reduction (28\%) in genital warts in males has also been seen-a herd immunity effect. ${ }^{8}$ Perhaps with the recent phase 3 clinical trial data showing effectiveness of a quadrivalent vaccine in reducing genital warts in males, as well as HPV16/18-related anal intraepithelial neoplasia, the precursor lesion to anal cancer, a case for vaccinating boys as well as girls could ultimately translate into true declines in genital warts, as well as other HPV-related diseases worldwide. In addition, such an approach would take the stigma away from just vaccinating girls and would ultimately decrease HPV-related diseases in men who have sex with men, as well as reducing any potential influence that genital warts and/or HPV infection may have on HIV transmission.

Next in the time sequence of HPV-related diseases, one expects to see a reduction in incidence of abnormal Pap cytology in a vaccinated cohort. In a review of cervical Pap smear abnormalities recorded on the Victorian Cervical Cytology Registry, one of Australia's population-based Pap test registers, a modest and significant decrease in high-grade abnormalities was demonstrated in women aged $<18$ years ( $<16$ years at vaccination), during 2007-2009 when the HPV vaccination programme was delivered, compared with the prevaccination period. 9 We expect to see similar reduced changes in Pap abnormalities in those countries with high coverage of school-based programmes with the bivalent vaccine, such as the UK. Such changes will bring along other challenges, as, ultimately, reduction in cytology screening abnormalities will translate into a reduced positive predictive value for Pap cytology for abnormalities, requiring more sensitive assays for screening such as HPV DNA assays, with triage to cytology for those found positive for HPV infection. As the success of Pap screening programmes has relied on extensive education of clinicians as well as consumers, such potential changes in practice will need to be well articulated at all levels, reinforced, audited and modified to ensure effective outcomes.

Ultimately, and it will take decades given the natural history of HPV infection, a decline in HPVrelated cancers due to types 16 and 18 will be seen. But what does all this mean for the rest of the world, especially in low and middle income countries where much of the burden of disease is seen globally?

First, in considering the fact that the currently licensed cervical cancer vaccines are relatively new and aimed at preadolescent and adolescent girls (an age group not commonly targeted for vaccination), as well as being vaccines for prevention of diseases occurring years later, and the necessary infrastructure for vaccine rollout is lacking, we should take a step back and learn from hepatitis $B$ vaccination programmes. ${ }^{10}$ Inclusion of HPV vaccination in national immunisation programmes, possibly 'piggybacking' on the well-established WHO expanded programme on immunisation (EPI) for even the most resource-poor countries would be one way to achieve such a goal. This potential use of the 
well-established infrastructure already developed for the EPI could be used for adolescent programmes in those countries where school-based programmes are impractical.

Second, the expensive HPV vaccines pose a problem in affordability for the poorest countries, requiring other mechanisms for funding the setting up of HPV vaccine programmes. One is that of the GAVI Alliance (formerly the 'Global Alliance for Vaccines and Immunisation'), which currently funds vaccines for the 72 poorest nations worldwide. While GAVI has been a mechanism for introducing new vaccines, and the GAVI Board has endorsed the HPV vaccine in principle, funding for this to be implemented is still needed. Recently announced, and perhaps a step towards helping achieve sustainable public sector access in the most resource-scarce areas of the world, is the pledge by Merck to provide the quadrivalent vaccine to the GAVI Alliance at a price of US\$5.00 per dose. Although there are tiered prices for both HPV vaccines for some countries, with opportunities for a 'no-profit price', this is still out of the financial reach of many. Other mechanisms include collective buying potential of countries negotiating prices together such the Pan American Health Organization (PAHO) revolving fund whereby countries pay for vaccines obtained at very low prices and as negotiated by the PAHO. One could envisage a partnership, a win-win position for all if those in resource-poor countries with expertise in vaccine manufacturing could partner current vaccine manufacturers to ensure that high-quality vaccines can be produced with more efficient pricing structures. This may allow distribution, not only to females but also to males, which could be very appropriate given the morbidity men suffer from HPV, as well as their role in transmission of HPV to and from women. ${ }^{11}$

One recently reported novel partnership is that of the manufacturer of the quadrivalent vaccine, Merck (known as MSD outside the USA and Canada), with the Royal Government of Bhutan, where, with the endorsement of Bhutanese Royalty as well as the Australian Cancer Foundation (a charity whose mission is to minimise the incidence and burden of cervical cancer and is supporting the national vaccination programme by providing financial support to the government of Bhutan to secure doses of vaccine at the access price after the first year of the programme is complete), all young girls $12-18$ years of age are to be vaccinated. Bhutan therefore will be the first lowincome country in the world to implement a national vaccination programme with an HPV cervical cancer vaccine.

Ultimately, whether a country chooses to implement an HPV vaccine will depend not only on cost, but also on the excellent efficacy, immunogenicity and safety that both licensed vaccines give to 16/18-related disease. How much they value 6/11-related disease coverage over this will determine their programme.

\section{CONCLUSION}

If we are to see a worldwide reduction in HPV-related neoplastic disease, it is imperative that many groups work together to achieve the common goal of the poorest nations worldwide gaining access to these highly effective prophylactic cervical

\section{Key messages}

There are two highly efficacious, immunogenic, human papillomavirus (HPV) vaccines licensed and available to prevent HPV16/18-related disease, in those naïve from infection.

- In real-life settings, reductions in genital warts have already been seen where the quadrivalent vaccine has been used in comprehensive public health programmes.

- Globally, there is a need for greater access (cheaper prices plus efficient infrastructure processes) to really affect HPVrelated diseases.

cancer vaccines to reduce the burden of disease from this leading cancer in women, and prevent HPV-related disease in men as well. Currently, there is only one vaccine that targets genital warts. In the future, multivalent vaccines will allow wider HPV genotype coverage and prevent more disease.

Funding SMG has received advisory board fees and grant support from CSL and GlaxoSmithKline, and lecture fees from Merck, GSK and Sanofi Pasteur. In addition, SMG has also received funding through her institution to conduct HPV vaccine studies for MSD and GSK.

Competing interests SMG is a member of the Merck Global Advisory Board as well as the Merck Scientific Advisory Committee for HPV.

Contributors SMG is the sole author of this article, the content of which is the opinion entirely of this author.

Provenance and peer review Commissioned; externally peer reviewed.

\section{REFERENCES}

1. Zur Hausen H. Human papillomaviruses and their possible role in squamous cell carcinomas. Curr Top Microbiol Immunol 1977;78:1-30.

2. Wallboomers JM, Jacobs MV, Manos MM, et al. Human papillomavirus is a necessary cause of invasive cervical cancer worldwide. J Pathol 1999;189:12-19.

3. Garland SM, Steben M, Sings H, et al. Natural history of genital warts: Analysis of the placebo arm of two randomized phase iii trials of a quadrivalent HPV (types 6, 11 16, 18) vaccine. J Infect Dis 2009;199:805-14.

4. Kjær SK, Tran TN, Sparen P, et al. The Burden of Genital Warts: A Study of nearly 70,000 women from the general female population in the 4 nordic countries. $J$ Infect Dis 2007:196:1447-54.

5. Paavonen J, Naud P, Salmeron J, et al. Efficacy of human papillomavirus (HPV)-16/ 18 ASO4-adjuvanted vaccine against cervical infection and precancer caused by oncogenic HPV types (PATRICIA): final analysis of a double-blind, randomised study in young women. Lancet 2009;374:301-14.

6. Garland SM, Hernandez AM, Wheeler CM, et al. Quadrivalent vaccine against human papillomavirus to prevent anogenital diseases. $N$ Engl J Med 2007;356:1928-43

7. FUTURE II Study Group. Quadrivalent vaccine against human papillomavirus to prevent high-grade cervical lesions. N Engl J Med 2007;356:1915-27.

8. Donovan B, Franklin N, Guy R, et al. Quadrivalent human papillomavirus vaccination and trends in genital warts in Australia: analysis of national sentinel surveillance data. Lancet Infect Dis 2010;11:39-44.

9. Brotherton JML, Fridman M, May CL, et al. Early eff ect of the HPV vaccination programme on cervical abnormalities in Victoria, Australia: an ecological study. Lancet 2011:377:2085-92.

10. Heffernan ME, Garland SM, Kane MA. Global reduction of cervical cancer with human papillomavirus vaccines: insights from the hepatitis B virus vaccine experience. Sex health 2010;7:383-90.

11. Garland SM. Prevention strategies against human papillomavirus in males. Gynecol Oncol 2010;117:S20-5. 